\title{
NO TRUMP!: A statistical exercise in priming ${ }^{1}$
}

\author{
Jonathan Falk and Andrew Gelman ${ }^{2}$
}

15 June 2016

\begin{abstract}
How are people unconsciously influenced by the rise of Donald Trump? We test the theory that Trump's rise has irrationally changed the behavior of one group of people: elite bridge players, whom we assume are otherwise completely typical. We examine the hands played in one of the premier North American bridge events, the Vanderbilt Knockout Tournament, in 1999 and 2015. We find that players had significantly higher probabilities of making No Trump contracts in the 2015 period compared with the earlier periods. We conclude that in the latter period, defending players are subtly deranged by the prospect of Trump and play their hands worse. By contrast, a 2015 European tournament shows no significant difference with the earlier 1999 tournament. This strengthens our conclusion.
\end{abstract}

Hypothesis: Many studies have demonstrated that people can be unconsciously goaded into different behavior through subtle psychological priming. ${ }^{3}$ We investigate the effect of the prospect of a Donald Trump presidency on the behavior of the top level of American bridge players.

Data: We have downloaded a number of tournaments ${ }^{4}$ from the bridge database at http://bridgetoernooi.com/. Of these, the most recent US tournament available is the 2015 Vanderbilt Knockout Teams. While this tournament was played in March 2015, and Donald Trump did not formally announce his candidacy until June, speculation about a Trump candidacy was certainly prevalent. If anything, this makes our results conservative, and we would expect even stronger effects as his path to the nomination gained strength.

We use two different controls: the Vanderbilt Knockout tournament results from 1999, five years before Donald Trump even had a reality show, and the Dutch Team Championship of 2015. If our hypothesis is correct, No Trump contracts should be more successful in the 2015 Vanderbilt Knockouts than in the same tournament played in 1999. Contemporaneous Dutch matches should show no significant differences from the earlier Vanderbilt matches. We expect the direction of the Trump effect to be negative (that is, to the benefit of No Trump contracts) given the association of the game of bridge with the Democratic party. ${ }^{5}$

\footnotetext{
${ }^{1}$ We were going to publish this in PLOS-One, but we didn't want to pay the $\$ 1495$.

${ }^{2}$ Please do not tell our employers that we spent any time doing this.

${ }^{3}$ There are of course famous examples, like the Florida Priming effect of Bargh, Chen and Burroughs (1996) but we prefer the cleaner 50 Shades of Gray effect (Nosek, Spies and Motyl, 2012).

${ }^{4}$ We have downloaded lots more tournaments than those reported here. Future papers will address these additional datasets.

${ }^{5}$ Consider, for example, the liberal super PAC American Bridge, or the labeling of liberal senator Elizabeth Warren as playing a "key bridge role" for her party (Rachel Maddow Show, 2016).
}

Presented at the $33^{\text {rd }}$ International Conference on Machine Learning, Workshop on Data-Efficient Machine Learning, New York City. ～Embargoed until Friday 24 June 2016, 10:30am EDT 
Results: The following table gives the summary results from these three tournaments: ${ }^{6}$

\begin{tabular}{|l|c|c|c|}
\hline & Vanderbilt 2015 & Vanderbilt 1999 & Dutch 2015 \\
\hline Hands Played & 781 & 205 & 127 \\
\hline $\begin{array}{l}\text { Percentage Played in No Trump } \\
\text { E(NT) }\end{array}$ & $28.81 \%$ & $25.98 \%$ & $25.98 \%$ \\
\hline $\begin{array}{l}\text { Percentage of "Made" No Trump } \\
\text { Hands E(NTxM) }\end{array}$ & $19.97 \%$ & $12.60 \%$ & $18.63 \%$ \\
\hline Conditional Success Rate E(M|NT) & $69.32 \%$ & $48.50 \%$ & $71.71 \%$ \\
\hline
\end{tabular}

The rate of hands played in No Trump was identical to four decimal places in the 1999 U.S. and 2015 Dutch matches and was sharply higher in the 2015 U.S. match, as predicted by the theory that recent political developments have made American bridge players more partial to No Trump contracts. With a one-sided p-value of 0.17 , this difference does not quite reach traditional levels of statistical significance, implying that this is a small effect.

When we look at the percentage of No Trump contracts made, the Vanderbilt 2015 tournament is significantly higher than the Vanderbilt 1999 tournament. The t-test yields a p-value of 0.0492, easily passing the traditional significance level of 5 percent. By contrast, however, we cannot conclude that the Dutch tournament is any different in this regard than the earlier U.S. tournament (recall that their rates of No Trump play were identical). The p-value for a difference in the Dutch and earlier U.S. success rates of No Trump hands is well above any significance threshold. Further, since the Dutch made rate is lower than the Vanderbilt 2015 rate, we cannot reject the possibility that the subliminal influence of Trump even managed to reach the Netherlands. This possible contamination should help explain why the U.S. and Dutch results from 2015 are only marginally significantly different from each other.

We note a possible anomaly in the conditional success rate, i.e. the fraction of No Trump hands successfully made. The Dutch results are actually slightly higher than the 2015 Vanderbilt results, though not significantly so. The startling result is how poorly the U.S. 1999 declaring sides did, making less than 50 percent of their No Trump hands. This can be explained (after thinking about it over a couple of beers) by the fact that back in 1999 one could afford to be nonchalant about declaring No Trump since the decision to do so had no overt political implications; in 2015, if you're going to state the word "Trump" aloud, you need extra confidence that you can succeed, therefore inoculating yourself from a charge of political bias.

As is often the case, priming effects are subtle, unexpected, and newsworthy, while at the same time being perfectly coherent with theory.

\footnotetext{
${ }^{6}$ We plan to release the dataset after we have published this research in five different journals (B. Frey, passim). In the meantime, we are not concerned with any data transcription errors since they would simply lead to attenuation bias which would make our results even stronger (Reinhart and Rogoff, 2010).
} 
Discussion: These disturbing phenomena seem to deny all our usual scientific ideas. How we should like to discredit them! Unfortunately the statistical evidence is overwhelming. ${ }^{7}$

Trumpmania appears to cause a slight increase in the fraction of games played at No Trump. This suggests either that elite players are more likely to issue the phrase "No Trump" when bidding, or that the phrase stated by their opponents stuns them into silence. ${ }^{8}$ While this effect is suggestive, it would require larger sample sizes to definitively confirm.

When No Trump hands are played, however, the declaring players do significantly better in 2015 than in 1999. Whether this is due to better (perhaps more aggressive and demeaning) play by declarers or mistakes by defenders cannot be determined without further study, perhaps on a sample of 24 undergraduate psychology students and confirmed on 100 Mechanical Turk participants.

It would be natural to attribute the findings of this paper to a direct response to the candidacy of Donald Trump; however we must consider other pathways as well, as it is well understood from social psychology that seemingly trivial inputs such as football games, and subliminal images, and shark attacks ${ }^{9}$ can be more important then actual policy positions when affecting political attitudes. An example is the well-established finding that visual contrast polarizes moral judgment (Zarkadi and Schnall, 2013), which was later reinterpreted in terms of latent associations of chessboard patterns with Russia, and thus foreign policy (Gelman, 2016).

In the present example, the relevant indirect pathway comes through the well-established principle of embodied cognition (e.g., Bargh, Chen, and Burrows, 1996). In the political context the word Bridge triggers immediate association with the recent "Bridgegate" scandal in New Jersey, ${ }^{10}$ a sequence of events closely tied to Chris Christie, the New Jersey governor, former presidential candidate, possible future Secretary of Transportation, ${ }^{11}$ and the first major Republican leader to endorse Donald Trump (e.g., Diamond et al., 2016). This pathway could be considered more of a moderator than a mediator in that bridge players will likely be aware of bridge-related news and hence particularly attuned to the Trump candidacy. ${ }^{12}$

We are unfortunately unable to separate the effect of Trump specifically from the fact that each bridge hand is called a "deal," which obviously will prime for Mr. Trump's best-known

\footnotetext{
${ }^{7}$ See Turing (1950), as quoted in Wagenmakers et al. (2015).

${ }^{8}$ At these tournaments no one actually speaks, using cards for the bids instead, but the point is unchanged.

${ }^{9}$ No kidding. See Healy and Malhotra (2010), Gelman (2015), Achen and Bartels (2012).

${ }^{10}$ An informal survey of leading bridge players finds that the vast majority live in New Jersey, or are from New Jersey, or have been to New Jersey, or know someone who has been to New Jersey.

${ }^{11}$ This is a joke. Chris Christie will never be Secretary of Transportation.

12 One might pedantically object that Christie did not endorse Trump until February, 2016, nearly a year after the 2015 data analyzed in our paper. We respond to this objection by noting two peer-reviewed publications that demonstrate statistically significant causality going backward in time: Witztum, Rips, and Rosenberg (1994) and Bem (2011).
} 
publishing effort, Trump: The Art of the Deal (1987), or for the association of bridge "hands" with the presidential candidate's notoriously foreshortened digits.

Relation to future work: We expect that after these results appear in NPR, TED, Gladwell, and the prestigious Proceedings of the National Academy of Sciences, there will be pushback from the inevitable replication bullies, those uncreative types who seem to exist only to criticize.

To save everyone trouble, we will preregister now the following responses to any future failed replications: (1) The replication was unfaithful to our original study because of various details not mentioned in this publication because of lack of space; (2) The replication was successful in demonstrating a heretofore unhypothesized interaction with outdoor temperature, relationship status, parental socioeconomic status, or some other crucial variable not included in our original study; $\operatorname{and}^{13}$ (4) Had the replication used a large enough sample size, it would surely have been statistically significant.

In short, disbelief is not an option. The results are not made up, nor are they statistical flukes. You have no choice but to accept that the major conclusions of these studies are true. ${ }^{14}$

Technical note: We originally wrote this note in Latex, but then we converted it to Word to make it more accessible. ${ }^{15}$

\section{Bibliography}

Achen, C. H., and Bartels, L. M. (2012). Blind retrospection: Why shark attacks are bad for democracy. Working paper, Vanderbilt University.

Bargh, J. A., Chen, M., and Burrows, L. (1996). Automaticity of social behavior: Direct effects of trait construct and stereotype activation on action. Journal of Personality and Social Psychology, 71, 230-244.

Bem, D. J. (2011). Feeling the future: Experimental evidence for anomalous retroactive influences on cognition and affect. Journal of Personality and Social Psychology, 100, 407-425.

Diamond, J., Tapper, J., Mattingly, P., and Collinson, S. (2016). Chris Christie endorses Donald Trump. cnn.com, 26 Feb. http://www.cnn.com/2016/02/26/politics/chris-christie-endorsesdonald-trump/

Fowler, A., and Montagnes, B. P. (2015). College football, elections, and false-positive results in observational research. Proceedings of the National Academy of Sciences, 112, 13800-13804.

\footnotetext{
${ }^{13}$ Point (3) was removed to save space, even though this footnote explaining that decision takes up more space than the original point (3).

${ }^{14}$ See Kahneman (2011), as quoted in Wagenmakers et al. (2011).

${ }^{15} \mathrm{Cf}$. Technical note of Gelman (2010).
} 
Gelman, A. (2010). "How many zombies do you know?": Using indirect survey methods to measure alien attacks and outbreaks of the undead. http://arxiv.org/abs/1003.6087

Gelman, A. (2015). Disagreements about the strength of evidence. Chance.

Gelman, A. (2016). 64 shades of gray: The subtle effect of chessboard images on foreign policy polarization. Statistical Modeling, Causal Inference, and Social Science blog, 27 Apr. http://andrewgelman.com/2016/04/27/51-shades-of-gray/

Healy, A, and Malhotra, N. (2010). Random events, economic losses, and retrospective voting: Implications for democratic competence. Quarterly Journal of Political Science, 5, 193-208.

Kahneman, D. (2011). Thinking, Fast and Slow. London: Allen Lane.

Nosek, B., Spies, J. R., and Motyl, M. (2012). Scientific utopia: II. Restructuring incentives and practices to promote truth over publishability. Perspectives on Psychological Science, 7, 615631.

Rachel Maddow Show (2016). Warren plays key bridge role for Democrats, 9 June. http://www.msnbc.com/rachel-maddow/watch/warren-plays-key-bridge-role-for-democrats702716995524

Sunn Classic Pictures (1977). In Search of Noah's Ark. ${ }^{16}$

Trump, D., and Schwartz, T. (1987). Trump: The Art of the Deal. New York: Warner Books.

Turing, A. M. (1950). Computing machinery and intelligence. Mind, 59, 433-460.

Von Daniken, E. (1968). Chariots of the Gods? ${ }^{17}$

Wagenmakers, E. J., Wetzels, R., Borsboom, D., Kievit, R., and van der Maas, H. L. J. (2015). A skeptical eye on psi. In May, E., and Marwaha, S. (Eds.), Extrasensory Perception: Support, Skepticism, and Science, 153-176. ABC-CLIO.

Witztum, D., Rips, E., and Rosenberg, Y. (1994). Equidistant letter sequences in the book of Genesis. Statistical Science, 9, 429-438.

Zarkadi, T., and Schnall, S. (2013). "Black and White" thinking: Visual contrast polarizes moral judgment. Journal of Experimental Social Psychology, 49, 255-359.

\footnotetext{
${ }^{16}$ This paper was not mentioned in the article but it seemed relevant so we threw it into the bibliography.

${ }^{17}$ This one too.
} 